\title{
The Native and Denatured States of Ovalbumin
}

\author{
By Aftab A. Ansari, R. Ahmad and A. Salahuddin \\ Department of Biochemistry, Jawaharlal Nehru Medical College, Aligarh Muslim University, Aligarh, India
}

(Received 8 November 1971)

The conformational state of the product of protein denaturation caused by extreme $\mathrm{pH}$, heat, urea and guanidinium chloride had not been systematically investigated until 1967, when Tanford and coworkers demonstrated that proteins are fully denatured in $6 \mathrm{M}$-guanidinium chloride and probably in 8-10M-urea and exist as random or cross-linked random coils. The completeness of urea denaturation has been demonstrated in only a few cases (see Tanford, 1968). It would therefore be worth while to test whether or not the urea-denatured state of a protein is the same as the one obtained in $6 \mathrm{M}$ guanidinium chloride. Despite systematic studies by Frensdorff et al. (1953) (see also Simpson \& Kauzmann, 1953) on urea denaturation of ovalbumin, the nature of the conformational state of urea-denatured ovalbumin remained uncharacterized. In the present communication we report our viscosity results on native ovalbumin and its denatured state obtained in $10 \mathrm{M}$-urea and in $6 \mathrm{M}$-guanidinium chloride $+0.1 \mathrm{M}$ 2-mercaptoethanol.

For viscosity measurements at $35 \pm 0.05^{\circ} \mathrm{C}$ the concentration of ovalbumin (grade V; Sigma Chemical Co., St. Louis, Mo., U.S.A.), c, in $\mathrm{g} / \mathrm{ml}$, was determined by the dry-weight method by taking isoionic protein solutions prepared by the Dintzis (1952) method in weighing bottles and heating them at $105-107^{\circ} \mathrm{C}$ to constant weight. Calibrated CannonFenske viscometers with flow times of 98.9 and $384.6 \mathrm{~s}$ for distilled water at $35^{\circ} \mathrm{C}$ were used. For viscometers having time of fall greater than 200 s the correction due to kinetic energy, if any, would be negligible. The time of fall of solvent, $t_{0}$, and that of protein solution, $t$, in the viscometers were measured within $\pm 0.05 \%$, by a stopwatch reading to $0.1 \mathrm{~s}$. The density of solvent, $\rho_{0}$, was determined by the standard procedure. The intrinsic viscosity, $[\eta]$, of ovalbumin was computed from a plot of $\left(t-t_{0}\right) / t_{0} \cdot c$ versus $c$ by using the expression (Tanford, 1955):

$$
\begin{aligned}
& {[\eta]=\lim _{c \rightarrow 0}\left(\eta-\eta_{0}\right) / \eta_{0} \cdot c=} \\
& \lim _{c \rightarrow 0}\left(t-t_{0}\right) / t_{0} \cdot c+\left(1-\bar{v}_{2} \cdot \rho_{0}\right) / \rho_{0}
\end{aligned}
$$

where $\eta_{0}$ and $\eta$ refer to the viscosity of solvent and that of solution, and $\bar{v}_{2}$ is the partial specific volume of the protein and was taken to be $0.75 \mathrm{ml} / \mathrm{g}$ (Neuberger \& Marshall, 1966). The density of $0.1 \mathrm{M}$ phosphate buffer, pH6.5, was found to be $1.0034 \mathrm{~g} /$ ml. The results are shown graphically in Fig. 1, in which the three lines were drawn by the method of least squares. The value of $[\eta]$ for native ovalbumin in phosphate buffer, $\mathrm{pH} 6.5$ and $I 0.42$, at $35 \pm 0.05^{\circ} \mathrm{C}$ was calculated to be $3.55 \mathrm{ml} / \mathrm{g}$, which strongly suggests that the native ovalbumin molecule is fairly compact and globular. The intrinsic viscosity of a protein can also be expressed in terms of partial specific volumes (Tanford, 1961):

$$
[\eta]=\nu\left(\bar{v}_{2}+\sum m_{i} \cdot v_{i}^{0}\right)
$$

in which $\nu$ is the Simha's or shape factor and is 2.5 for spheres and larger for ellipsoids, and $m_{t}$ is the $\mathrm{g}$ of a solvent component with partial specific volume $v_{i}^{0}$ bound to $1 \mathrm{~g}$ dry wt. of protein. If the native ovalbumin in the phosphate buffer binds only water, for which $v_{\imath}^{0}$ may be taken to be 1 , the maximum amount of water bound to $1 \mathrm{~g}$ of protein may be calculated by setting $\nu=2.5$ in eqn. (2). Thus the value of $m_{t}$ turns out to be $0.67 \mathrm{~g} / \mathrm{g}$ of protein. The maximum value of $\nu$ showing maximum asymmetry of the ovalbumin

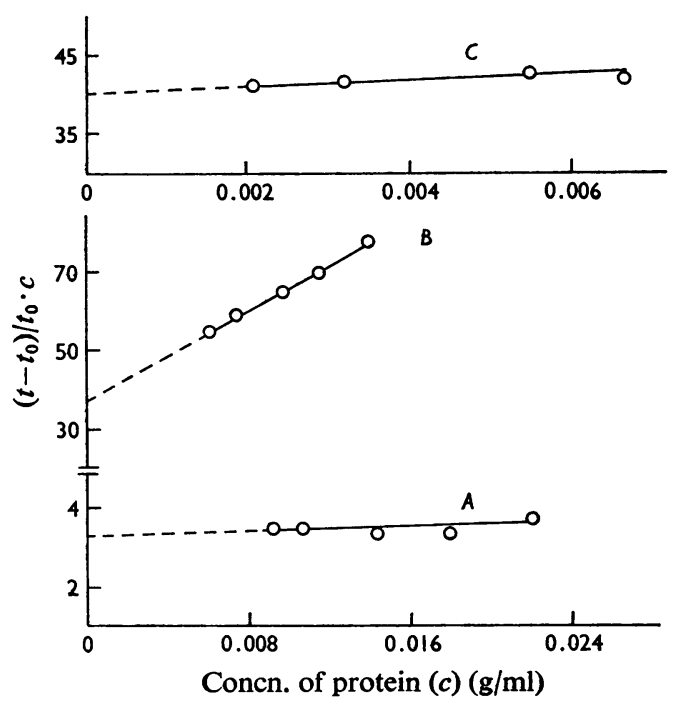

Fig. 1. Viscosity data for native and denatured ovalbumin

Curve $A$ represents the plot for ovalbumin at pH6.5 and $I 0.42$, at $35^{\circ} \mathrm{C}$. Curves $B$ and $C$ represent similar plots in $10 \mathrm{M}$-urea, pH6.5 and $I 0.49$, and in $6 \mathrm{M}$-guanidinium chloride $+0.1 \mathrm{M}$-2-mercaptoethanol, pH5.1, respectively. 
molecule, calculated by eqn. (2) by using $\sum m_{l} \cdot v_{i}^{0}=0$, turns out to be 4.7 , which corresponds to an axial ratio, $a / b$, of 4 for the prolate ellipsoid. The two extreme behaviours probably do not represent the true hydrodynamic behaviour of the protein. In fact, Bull \& Breese (1970) have shown that $1 \mathrm{~g}$ of ovalbumin binds about $0.29 \mathrm{~g}$ of water in the absence of electrolyte, so that in the presence of electrolyte, sodium phosphate, the binding of water by ovalbumin nay be even less. By using the value of $0.29 \mathrm{~g}$ for $m_{l}$ in eqn. (2) the values of $\nu$ and $a / b$ were calculated to be 3.4 and 2.7 respectively. This, together with the known frictional ratio, $f / f_{\text {min. }}$, of 1.17 (Tanford, 1961) for ovalbumin, indicates the compact and globular nature of ovalbumin. The equivalent hydrodynamic sphere calculated from viscosity data turns out to be $2.9 \mathrm{~nm}$ ( $(29 \AA)$ for molecular weight, $M$, equal to 43000 .

It may be pointed out that a given ovalbumin preparation may consist of three electrophoretically separable components, $A_{1}, A_{2}$ and $A_{3}$, having respectively two, one and no phospho groups. The three components of ovalbumin were, however, found to be indistinguishable in the ultracentrifuge (Tanford, 1961) and are similar in immunological behaviour (Durieux \& Kamiski, 1956; A. A. Ansari $\&$ A. Salahuddin, unpublished work). It can therefore be reasonably assumed that the presence or absence of phospho groups does not affect the hydrodynamic behaviour of ovalbumin. Further, at a relatively higher ionic strength of 0.42 , the presence of charge on the protein molecule contributed by phospho groups will not affect the value of the intrinsic viscosity of the protein.

By using 1.0907 and $1.4470 \mathrm{~g} / \mathrm{ml}$ for $\rho_{0}$ in $10 \mathrm{M}$-urea and in $6 \mathrm{M}$-guanidinium chloride +2 -mercaptoethanol, the values of intrinsic viscosity of ovalbumin in $10 \mathrm{M}$ urea and in $6 \mathrm{M}$-guanidinium chloride $+0.1 \mathrm{M}-2$ mercaptoethanol were computed by setting $\bar{v}_{2}=0.75$, from the curves $B$ and $C$ of Fig. 1, and were found to be 37.9 and $39.9 \mathrm{ml} / \mathrm{g}$ respectively. A small discrepancy in the value of $[\eta]$ may very well be due to a solvent effect. The intrinsic viscosities of several reduced proteins have been found (Tanford, 1968) to be lower in $8 \mathrm{M}$-urea than in $6 \mathrm{M}$-guanidinium chloride. For example, the intrinsic viscosity of reduced $\beta$-lactoglobulin in 8 $\mathrm{M}$-urea, in which the random-coil nature of the protein had been clearly demonstrated (Pace \& Tanford, 1968), was found to be $21.6 \mathrm{ml} / \mathrm{g}$, which is more than $5 \%$ lower than that $(22.8 \mathrm{ml} / \mathrm{g})$ in 6M-guanidinium chloride.
From the dependence of $[\eta]$ of several randomly coiled proteins in $6 \mathrm{M}$-guanidinium chloride on the chain length and hence the number of amino acid residues, $n$, of a protein, Tanford (1968) found an empirical relationship: $[\eta] \cdot M_{0}=77 n^{0.666}$, in which $M_{0}=M / n$. By using values of 111.1 and 387 respectively for $M_{0}$ and $n$, the value of $[\eta]$ of ovalbumin in $6 \mathrm{M}$-guanidinium chloride $+0.1 \mathrm{M}$-2-mercaptoethanol was calculated to be $36.7 \mathrm{ml} / \mathrm{g}$, which is about $8 \%$ lower than the experimental value. The root-meansquare end-to-end distance, $\left\langle\boldsymbol{L}^{2}\right\rangle^{\frac{1}{t}}$, calculated from viscosity data by using the appropriate equation (Tanford, 1968), was calculated to be $20.1 \mathrm{~nm}$ (201 $\AA$ ). From the amino acid composition the value of the unperturbed dimension, $\left\langle L_{2}\right\rangle^{ \pm}$, of randomly coiled ovalbumin was computed by the empirical equation of Tanford (1968) to be $16.5-18.1 \mathrm{~nm}$ (165-181 $\AA$ ). Thus the unperturbed end-to-end distance is $10-18 \%$ lower than the actual one calculated from the viscosity data. Such discrepancies have also been noted for other proteins in the random-coil state (Tanford, 1968). We therefore conclude that native ovalbumin, which is a fairly compact and globular molecule, exists as a random coil devoid of any native structure in $6 \mathrm{M}$-guanidinium chloride+ $0.1 \mathrm{M}-2$-mercaptoethanol. The protein probably exists in the same conformational state in $10 \mathrm{M}$-urea, where one or two disulphide bonds/molecule (Neuberger \& Marshall, 1966; Fothergill \& Fothergill, 1970) remain intact. The presence of disulphide bonds does not seem to impose any detectable physical constraint affecting the hydrodynamic volume.

Bull, H. B. \& Breese, K. (1970) Arch. Biochem. Biophys. 137, 299

Dintzis, H. (1952) Ph.D. Thesis, Harvard University

Durieux, J. \& Kamiski, M. (1956) Bull. Soc. Chim. Biol. 38, 1445

Fothergill, L. A. \& Fothergill, J. E. (1970) Biochem.J. 116, 555

Frensdorff, W. K., Watson, M. T. \& Kauzmann, W. (1953) J. Amer. Chem. Soc. 75, 5157

Neuberger, A. \& Marshall, R. D. (1966) in Glycoproteins (Gottschalk, A., ed.), chapter 11, p. 299, Elsevier, Amsterdam

Pace, N. C. \& Tanford, C. (1968) Biochemistry 7, 198

Simpson, R. B. \& Kauzmann, W. (1953) J. Amer. Chem. Soc. 75,5139

Tanford, C. (1955) J. Phys. Chem. 59, 798

Tanford, C. (1961) Physical Chemistry of Macromolecules, pp. 390-396, Wiley, New York

Tanford, C. (1968) Advan. Protein Chem. 23, 121 